Check for updates

Cite this: Mater. Adv., 2022, 3, 1071

Received 5th October 2021 Accepted 14th November 2021

DOI: $10.1039 / \mathrm{d} 1 \mathrm{ma} 00925 \mathrm{~g}$

rsc.li/materials-advances

\title{
Chalcogen-substituted PCBM derivatives as ternary components in PM6:Y6 solar cells $\dagger$
}

\author{
José G. Sánchez $\neq^{\mathrm{a}}$ Andrea Cabrera-Espinoza, $\ddagger^{\mathrm{b}}$ Eugenia Martínez-Ferrero, (D) ${ }^{a}$ \\ Juan Luis Delgado (D)*bc and Emilio Palomares (D)*ad
}

\begin{abstract}
Ternary organic solar cells (TOSCs) are a promising approach to enhance the power conversion efficiency in organic-based solar cells. The combination of fullerene and non-fullerene acceptors is employed to optimize the light absorption and phase separation for better charge dissociation and collection. Herein, we describe the synthesis and characterization of three chalcogen substituted PCBM derivatives with a 5-membered aromatic ring linked to the methyl ester position with the objective to study the relationship between interphase separation and power conversion efficiency. Therefore, the effects of furan, thiophene and selenophene in the PCBM derivatives (PCBFu, PCBTh and PCBSe, respectively) are investigated on the photovoltaic performance of ternary organic solar cells based on PM6:Y6. We observed that the addition of the PCBTh and PCBSe derivatives increases the short circuit current density and the fill factor pointing to the suppression of charge recombination. In addition, surface and thermal analysis confirms that the morphology is optimized in both cases, which implies that organized thin film nanomorphology is key for the suppression of carrier losses. The TOSC with PCBTh exhibited the highest power conversion efficiency among all the devices reaching $14.6 \%$.
\end{abstract}

\section{Introduction}

Organic solar cells (OSCs) are considered a potential costeffective clean energy product due to their great features such as light weight, broad absorption in the visible range, solutionprocessing, and the capability to fabricate large, semitransparent and flexible devices. ${ }^{1-3}$ Nowadays, single-junction OSCs have reached a power conversion efficiency (PCE) of over $18 \%$ under sun-simulated light irradiation under standard measurement conditions. ${ }^{4,5}$ One of the key factors to prepare efficient OSCs has been the development of the polymer electron donor PBDB-T and its derivatives (e.g. PBDB-TF, PBDB-T-SF, PBDB-Th to name a few). ${ }^{6,7}$ However, the solar to energy conversion efficiency of OSCs based on these electron donors mixed with the widely used fullerenes has been limited by the weak light absorption in the visible and near-infrared (NIR) regions of the fullerene acceptors. ${ }^{8-10}$ Thus, the

\footnotetext{
${ }^{a}$ Institute of Chemical Research of Catalonia-The Barcelona Institute of Science and Technology (ICIQ-BIST), Avda. Països Catalans, 16, Tarragona, E-43007, Spain. E-mail: epalomares@iciq.es

${ }^{b}$ POLYMAT, University of the Basque Country UPV/EHU, Avenida de Tolosa 72 , Donostia - San Sebastián 20018, Spain. E-mail: juanluis.delgado@polymat.eu ${ }^{c}$ Ikerbasque, Basque Foundation for Science, Bilbao 48013, Spain

${ }^{d}$ ICREA, Passeig Lluis Companys 23, Barcelona, E08010, Spain

$\dagger$ Electronic supplementary information (ESI) available: Synthetic procedure and characterization data. See DOI: $10.1039 / \mathrm{d} 1 \mathrm{ma} 00925 \mathrm{~g}$

\# Contributed equally to this work.
}

replacement of the fullerenes by non-fullerene acceptors such as ITIC-family, IDIC-family and more recently the Y-family with broader light absorption in the visible and NIR regions has allowed a rapid enhancement of PCE owing to the better harnessing of the solar energy. ${ }^{11-15}$ In this sense, the spark of great interest in OSCs has arisen from the good performance of devices based on the PBDB-T donor PM6 (poly[[4,8-bis[5-(2-ethylhexyl)-4-fluoro-2-thienyl] benzo [1,2-b:4,5- $\left.b^{\prime}\right]$ dithiophene-2,6-diyl]-2,5-thiophenediyl [5,7-bis(2ethylhexyl)-4,8-dioxo- $4 H, 8 H$-benzo [1,2-c:4,5-c' $]$ dithiophene-1,3-diyl]2,5-thiophenediyl], see Fig. S1a, ESI $\dagger$ ) and the Y-series acceptor Y6 (2,2'- $\quad\left(\left(2 Z, 2^{\prime} Z\right)-((12,13-b i s(2-e t h y l h e x y l)-3,9-d i u n d e c y l-12,13-d i h y d r o\right.$ $[1,2,5]$ thiadiazolo $[3,4-e]$ thieno $\left[2^{\prime \prime}, 3^{\prime \prime}: 4^{\prime}, 5^{\prime}\right]$ thieno $\left[2^{\prime}, 3^{\prime}: 4,5\right]$ pyrrolo $[3,2-g]$ thieno $\left[2^{\prime}, 3^{\prime}: 4,5\right]$ thieno $[3,2-b]$ indole-2,10-diyl) bis (methanylylidene)) bis(5,6-difluoro-3-oxo-2,3-dihydro-1 $H$-indene-2, 1-diylidene))dimalononitrile), see Fig. S1b, ESI + ), which surpassed the long-standing PCE bottleneck of $15 \%{ }^{16}$ and closes the gap with perovskite-based solar cells. Consequently, many approaches to improve the performance of PM6:Y6 OSCs have been reported in the literature. ${ }^{17,18}$ Among those, ternary OSCs (TOSCs, i.e. incorporating an extra donor or acceptor into the binary system) are one of the most promising strategies since the third component can complement the light absorption spectra of the host materials and positively intervene on the intermolecular charge transfer and transport. ${ }^{19-21}$ Fullerene acceptors (e.g. PC71BM) have been used as a third component to improve the charge transport and energy transfer of donor:non-fullerene acceptor binary systems. ${ }^{22,23}$ Currently, it has been demonstrated that adding $20 \%$ of $\mathrm{PC}_{71} \mathrm{BM}$ and 
PCBM (related to the PM6) into the PM6:Y6 system is the optimum amount to achieve higher performance of devices. ${ }^{24,25}$ Thereby, we focused on the design and synthesis of PCBM derivatives compatible with the molecular structure of PM6 and Y6 capable of improving the charge generation and extraction of PM6:Y6 to obtain more efficient TOSCs. The PCBM derivatives featuring a 5membered aromatic ring endowed with oxygen, sulphur or selenium chalcogen atoms (furan, thiophene and selenophene) could have an impact on the morphology of PM6:Y6, which may improve the performance of these devices. Several studies have reported before the impact of the non-fullerene acceptors containing furan, thiophene and selenophene molecules on the morphology and charge generation and extraction of OSCs. ${ }^{26-28}$ On the other hand, the thiophene derivative of the PCBM has been previously studied by other authors as an acceptor in binary systems in combination with poly-3-hexylthiophene ( $\mathrm{P} 3 \mathrm{HT}$ ) or as a ternary component in P3HT:PCBM where it demonstrated their effect in the optimization of the macrophase separation due to the decrease in the interfacial surface energy. ${ }^{29,30}$ Thiophene derivatives of the PCBM have been also reported as electron transport materials in perovskite-based solar cells. However, the perovskite passivation activity of the $S$ was not enough to increase the solar efficiency in comparison to the non-substituted PCBM molecule. ${ }^{31}$ To expand the study about the influence of sulphur in ternary organic solar cells to other chalcogens, in this study, we report the synthesis and characterization of PCBM substituted with furan (PCBFu), thiophene (PCBTh) or selenophene (PCBSe) as the third component in TOSCs based on PM6:Y6. The impact of the PCBM-derivatives on the photovoltaic parameters, recombination mechanism and morphology of TOSCs has been evaluated. Our results demonstrated that PCBTh and PCBSe have positive effects on the morphology and recombination of PM6:Y6, which result in more efficient TOSCs compared to binary OSCs. In the particular case of PCBFu, the furan ring leads to a higher recombination degree and leakage current, which limit the efficiency of TOSCs.

\section{Results and discussion}

\section{Synthesis and characterization of the PCBM derivatives}

Chalcogenophene-based fullerene derivatives PCBX (PCBFu, PCBTh and PCBSe) were synthesized following the procedure shown in Scheme 1. In brief, chalcogenophene-2-carboxaldehydes 1a-c were reduced to the corresponding alcohols $2 \mathrm{a}-\mathrm{c}$ with $\mathrm{NaBH}_{4}$. Subsequently, 3a-c were obtained by the Steglich esterification reaction with 4-benzoylbutyric acid. The obtained ketoesters were then subjected to condensation reactions with p-tosylhydrazide forming the desired tosylhydrazones TsHX (TsHSe, TsHTh and TsHFu), which were transformed in situ to the active diazo species by treatment with sodium methoxy in pyridine as the solvent. The [2+1] cycloadditions with $C_{60}$ were carried out by heating the reaction mixtures at $80{ }^{\circ} \mathrm{C}$ and quenched when the formation of polyadducts was evidenced by thin-layer chromatography (TLC). The conversion of $[5,6]$-open isomers to the $[6,6]$-closed methanofullerenes was accomplished by heating at $180{ }^{\circ} \mathrm{C}$. The chemical structures of the monoadducts were confirmed using NMR, FT-IR

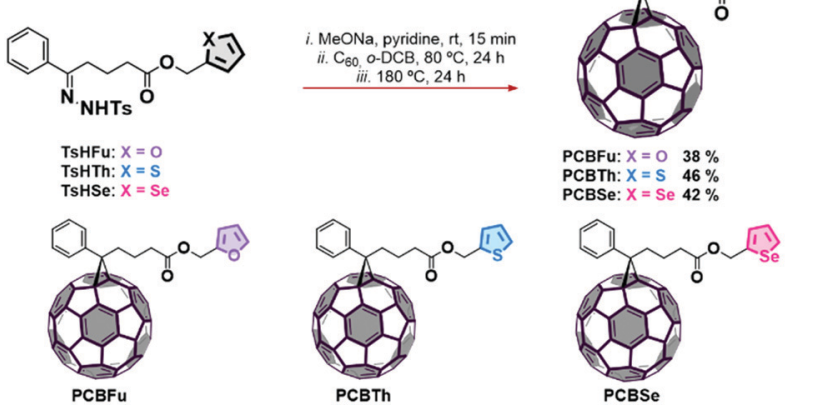

Scheme 1 Synthesis route and molecular structure of the PCBM derivatives PCBSe, PCBTh and PCBFu. Synthetic yields are indicated as percentages.

spectroscopy, and MALDI-TOF spectrometry (see the ESI $\dagger$, Fig. S3S27). The ${ }^{13} \mathrm{C}$ NMR spectra supported the structure of [6,6]-isomeric compounds, with the signal for the $\mathrm{C}(61)$ carbon (bridging carbon) appearing around $51.7 \mathrm{ppm}$ and the only $\mathrm{sp}^{3}$-hybridized carbon atom of the fullerene appearing at 79.5-79.6 ppm. In the FT-IR spectra of the target compounds (PCBSe, PCBTh and PCBFu), a strong characteristic band corresponding to $C_{60}$ appears at $c a$. $523 \mathrm{~cm}^{-1}$ and the bands at $1735 \mathrm{~cm}^{-1}$ and $1146 \mathrm{~cm}^{-1}$ reveal the presence of the ester moiety. The MALDI-TOF spectra of these compounds confirmed the presence of pure mono-substituted adducts, showing in each case the molecular ion peak at the appropriate $m / z$ values. Moreover, all the mass spectra are consistent with the simulated isotopic distribution. The LUMO and HOMO energy levels of the three PCBM derivatives were established by cyclic voltammetry (CV) (Fig. S2, ESI $\dagger$ ). The LUMO level of PCBMderivatives was estimated from the first onset reduction potential $\left(E_{\text {red }}^{I} v s . \mathrm{Fe} / \mathrm{Fe}^{+}\right)$using the equation $E_{\mathrm{LUMO}}=-e\left(E_{\mathrm{red}}^{I}+4.8\right)$, while the HOMO levels were calculated from $E_{\mathrm{HOMO}}=E_{\mathrm{LUMO}}-E_{\mathrm{g}}$, where $E \mathrm{~g}$ is estimated from the onset of the absorption. The LUMO value of PCBFu is quite similar to that of PCBM (-3.94 and $3.91 \mathrm{eV}$, respectively). PCBSe and PCBTh have lower LUMO values (3.98 and $4.03 \mathrm{eV}$, respectively) than that of PCBFu. The HOMO of three derivatives $(\mathbf{P C B F u}=-5.80 \mathrm{eV}, \mathbf{P C B T h}=-5.89 \mathrm{eV}$, and PCBSe $=-5.84 \mathrm{eV})$ is higher than that of PCBM $(-5.93 \mathrm{eV})$. Fig. 1 shows the energy level alignment for materials used in the devices. The energy levels for PEDOT:PSS, PM6, Y6, PCBM and PDINO $\left(N, N^{\prime}-\right.$ bis(N,N-dimethylpropan-1-amine oxide) perylene - 3,4,9,10tetracarboxylic diimide) were taken from the literature. ${ }^{16,32,33}$ All the optical and energetic properties of PCBM-derivatives are summarized in Table 1. It is worthy mentioning that the three derivatives have the same energy band gap $(1.86 \mathrm{eV})$, which is lower than that of PCBM $(2.02 \mathrm{eV})$. Therefore, the addition of chalcogens decreases the energy band gap in similar terms regardless of the nature of the substituting atom.

\section{Photovoltaic properties}

To assess the effect of PCBM derivatives on the PM6:Y6 based OSCs we fabricated devices following the conventional structure of ITO/PEDOT:PSS/PM6:Y6:PCB-X/PDINO/Al. Binary PM6:Y6 OSCs were also fabricated as control devices. 


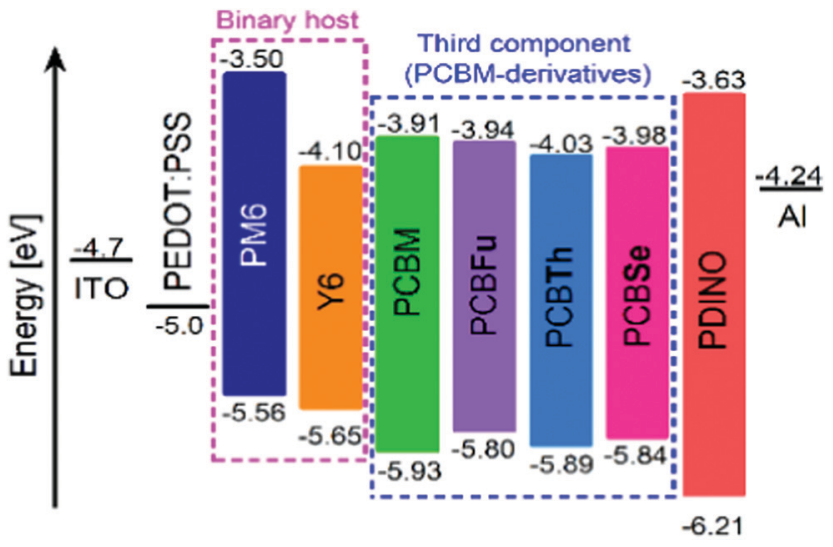

Fig. 1 Energy level alignment for PCBFu, PCBTh, and PCBSe and the materials used for the device fabrication. The energy levels for PEDOT:PSS, PM6, Y6, PCBM and PDINO were taken from the literature.

Table 1 Optical and energetics parameters of PCBM derivatives

\begin{tabular}{llllll}
\hline Compound & $\lambda_{\mathrm{abs}} \max [\mathrm{nm}]$ & $E_{g}{ }^{a}[\mathrm{eV}]$ & $E_{\mathrm{red}}^{I}[\mathrm{~V}]$ & $E_{\mathrm{LuMO}}{ }^{b}[\mathrm{eV}]$ & $E_{\text {номо }}{ }^{c}[\mathrm{eV}]$ \\
\hline PCBFu & 670 & 1.86 & -0.86 & -3.94 & -5.80 \\
PCBTh & 667 & 1.86 & -0.77 & -4.03 & -5.89 \\
PCBSe & 669 & 1.86 & -0.82 & -3.98 & -5.84
\end{tabular}

${ }^{a}$ Estimated from the onset wavelength $\left(\lambda_{\text {abs }}\right)$ of the UV-Vis spectra. ${ }^{b}$ Estimated from the first onset reduction potential $\left(E_{\text {red }}^{I} v s . \mathrm{Fe} / \mathrm{Fe}^{+}\right)$as $E_{\mathrm{LUMO}}=-e\left(E_{\mathrm{red}}^{I}+4.8\right) .{ }^{c}$ Calculated as $E_{\mathrm{HOMO}}=E_{\mathrm{LUMO}}-E_{\mathrm{g}}$.

The current density versus voltage $(J-V)$ characteristics of PM6:Y6 (1:1.2) and PM6:Y6:PCBM-derivatives (1:1:0.2) were measured under one-sun illumination (AM 1.5 G, $100 \mathrm{~mW} \mathrm{~cm}{ }^{-2}$ ). The $J-V$ characteristics of the best binary and the ternary systems are depicted in Fig. 2a. The photovoltaic parameters including short-circuit current-density $\left(J_{\mathrm{sc}}\right)$, opencircuit voltage $\left(V_{\mathrm{OC}}\right)$, fill factor $(\mathrm{FF})$ and power conversion efficiency (PCE) of all devices are listed in Table 2. The binary PM6:Y6 device shows a $V_{\mathrm{OC}}$ of $0.82 \mathrm{~V}$, a $J_{\mathrm{SC}}$ of $27.51 \mathrm{~mA} \mathrm{~cm}^{-2}$, a FF of 0.60 and a PCE of $13.42 \%$. As listed in Table 2, the TOSCs with commercial PCBM, and with PCBTh and PCBSe show slightly lower $J_{\mathrm{SC}}\left(26.86 \mathrm{~mA} \mathrm{~cm} \mathrm{~cm}^{-2}, 27.10 \mathrm{~mA} \mathrm{~cm} \mathrm{~cm}^{-2}\right.$ and $26.99 \mathrm{~mA} \mathrm{~cm}^{-2}$, respectively) than that of the binary OSCs. In contrast, the TOSC with PCBFu exhibits a much lower $J_{\mathrm{SC}}$ $\left(25.71 \mathrm{~mA} \mathrm{~cm}^{-2}\right)$ in comparison to the binary system.

As expected, the TOSC with PCBM shows the highest $V_{\mathrm{OC}}$ $(0.83 \mathrm{~V})$, while, the TOSC with PCBTh exhibits a similar $V_{\mathrm{OC}}$ $(0.82 \mathrm{~V})$ to that of the binary system. Interestingly, both TOSCs with PCBFu and PCBSe show a lower $V_{\mathrm{OC}}(0.80 \mathrm{~V}$ and $0.81 \mathrm{~V}$, respectively) than that of binary OSCs. Theoretically, the $V_{\mathrm{OC}}$ value can be estimated by the energy offset between the HOMO of the donor and the LUMO of the acceptor ${ }^{34}$ and thus the expected $V_{\mathrm{OC}}$ of TOSC-PCBFu and -PCBSe should be higher than that of the binary OSC, but lower than that of TOSCPCBM. These results suggest that the $V_{\mathrm{OC}}$ is limited by the loss mechanisms when adding PCBFu and PCBSe and discards the presence of alloyed states. ${ }^{35}$ It is noteworthy that all the TOSCs have higher FF compared to the binary device, with the TOSCPCBTh having the highest FF value. The improvement of FF is reflected in the increase of the PCE of most of the ternary devices, with TOSC-PCBTh having the highest value of $14.65 \%$, followed by TOSC-PCBM (14.36\%) and lastly the TOSC-PCBSe (13.97\%). Despite the TOSC-PCBFu exhibiting a better FF in comparison to the binary OSC, its PCE is lower (12.88\%), which is mainly limited by the simultaneous decrease of its $J_{\mathrm{SC}}$ and $V_{\mathrm{OC}}$.

The improvement of the FF may arise from the increase of the shunt resistance, which reduces the leakage current of the host system when a certain amount of PCBM derivatives is added. In order to confirm this hypothesis, the dark $J-V$ characteristics of binary and TOSCs were measured and are depicted in Fig. 2b. All the ternary devices, except PCBFu, have a lower leakage current than the binary one suggesting a decrease of charge recombination. ${ }^{36}$ In addition, the dark $J-V$ curves of TOSCs based on PCBM, PCBTh and PCBSe exhibited similar behaviour in the forward bias. On the other hand, those of both binary OSC and TOSC-PCBFu showed different behaviour in the forward range of $0.2-0.7 \mathrm{~V}$, which is typically associated with the recombination current region. ${ }^{37}$ To further analyse the variations in the $J_{\mathrm{SC}}$ of devices, external quantum efficiency (EQE) measurements were performed. Fig. 2c shows the EQE spectra and the integrated $J_{\mathrm{SC}}$ of the binary OSC and ternary devices. The calculated $J_{\mathrm{SC}}$ values from the integration of the EQE spectra corroborate those calculated from $J-V$ curves within a $\sim 5 \%$ mismatch (see Table 2).

The lower $J_{\mathrm{SC}}$ of TOSC-PCBFu can be ascribed to its lower EQE compared to the other devices. At this point, the lower $J_{\mathrm{SC}}$ and $\mathrm{EQE}$ of the TOSC with PCBFu cannot be attributed to the noncomplementary absorption spectra since the absorption spectrum of PM6:Y6:PCBFu is quite similar to those of other ternary systems (Fig. 2d). On the other hand, the TOSC-PCBTh has shown a higher intensity of EQE in the absorption range of 300-450 nm compared to the binary system, which agrees with the UV-Vis spectra. This behaviour is not observed for TOSCs based on PCBM and PCBSe, although in both cases there is complementary photon absorption in the $300-400 \mathrm{~nm}$ region. Moreover, the higher $J_{\mathrm{SC}}$ of the binary OSC results from its higher EQE because of stronger absorption in the range of $650-850 \mathrm{~nm}$ as observed in the UV-Vis spectrum (Fig. 2d). The lower absorption of all the TOSC series in this range comes from the lower ratio of the $\mathrm{Y} 6$ acceptor in ternary blends compared to the binary blend (1 and 1.2, respectively). These results demonstrate that PCBM-derivatives do not contribute to the photon absorption, and consequently to the $J_{\mathrm{SC}}$ improvement in TOSCs.

\section{Light intensity dependence of $\boldsymbol{J}-\boldsymbol{V}$ characteristics}

The analysis of $J-V$ characteristics as a function of light intensity is a common approach to assess the recombination mechanisms in photovoltaic devices. Fig. 3a shows the dependence of $J_{\mathrm{SC}}$ and $V_{\mathrm{OC}}$ against different light intensities $\left(P_{\text {light }}\right)$. The variation of $J_{\mathrm{SC}} v \mathcal{s}$. $P_{\text {light }}$ is fitted by the power-law function $J_{\mathrm{SC}} \propto P_{\text {light }}{ }^{\alpha}$ (Fig. 3a), where $\alpha$ represents the bimolecular recombination degree. The $\alpha$ value close to unity indicates that the bimolecular recombination is negligible and a limited space charge is not present under short-circuit 
a)

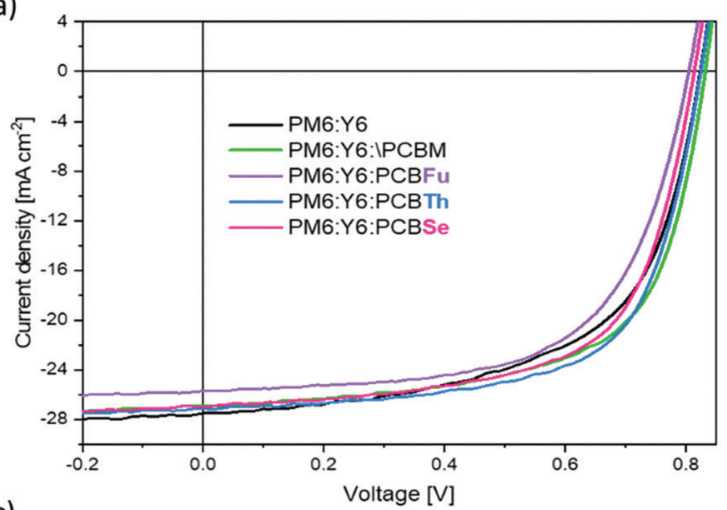

c)

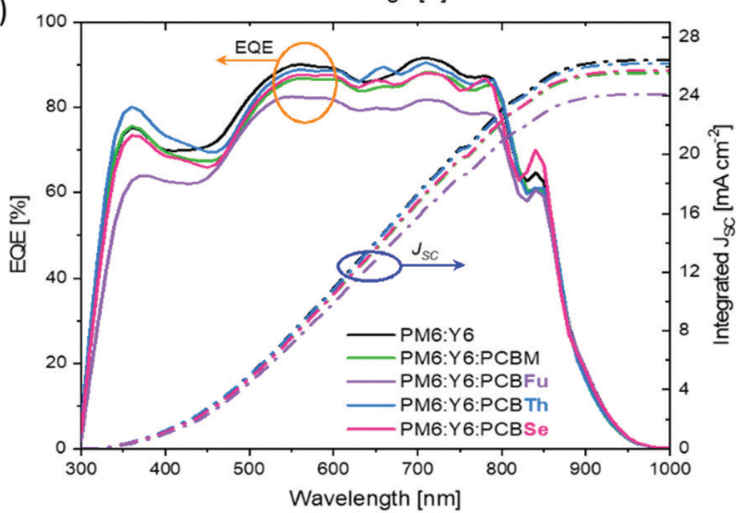

b)

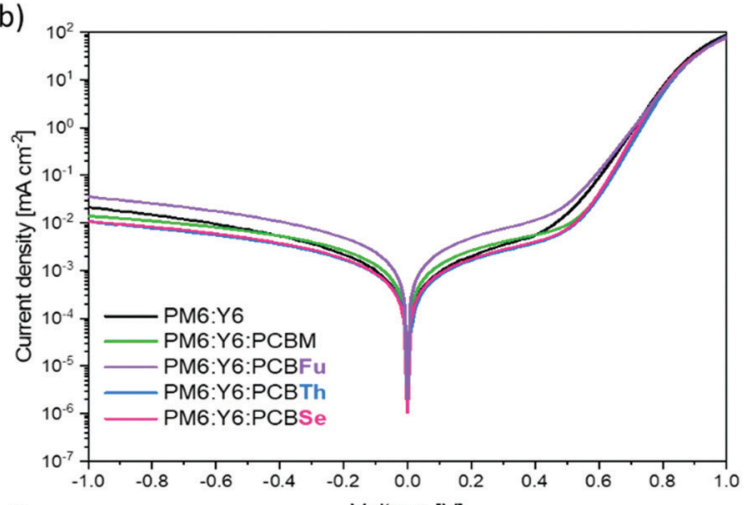

d)

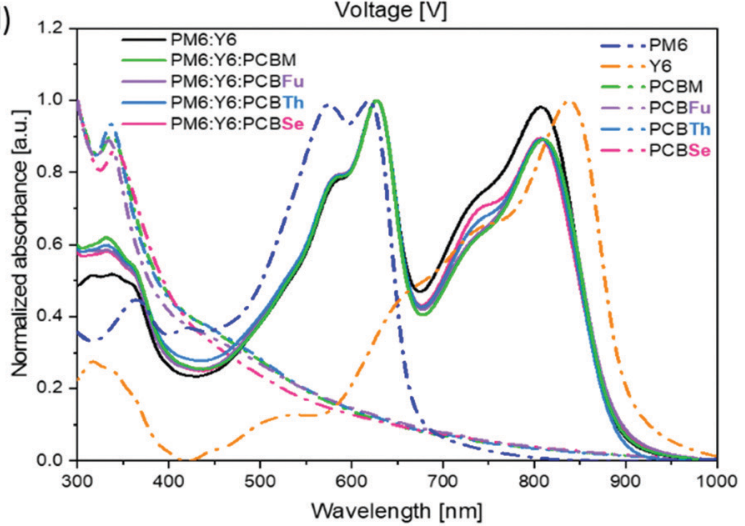

Fig. 2 Best current-density vs. voltage (J-V) characteristics under (a) simulated AM 1.5 G illumination and (b) dark conditions, and (c) EQE spectra (solid lines) and integrated $J_{S C}$ (dash dot lines) of binary OSCs and ternary OSCs with PCBM, PCBFu, PCBTh, and PCBSe. (d) Absorption spectra of neat PM6, Y6 and PCBM-derivatives (dash dot lines) and the blends, PM6:Y6:PCBM-derivatives (solid lines).

Table 2 Photovoltaic parameters of binary OSC (PM6:Y6) and ternary devices (PM6:Y6:PCBM-derivatives) recorded under AM 1.5 G illumination

\begin{tabular}{|c|c|c|c|c|c|}
\hline Device & $J_{\mathrm{SC}}\left[\mathrm{mA} \mathrm{cm}^{-2}\right]$ & Integrated $J_{\mathrm{SC}}{ }^{a}\left[\mathrm{~mA} \mathrm{~cm}^{-2}\right]$ & $V_{\mathrm{OC}}[\mathrm{V}]$ & FF & PCE $[\%]$ \\
\hline PM6:Y6:PCBM & $26.86(27.02)$ & 25.56 & $0.83(0.83)$ & $0.64(0.63)$ & $14.36(14.33)$ \\
\hline PM6:Y6:PCBFu & $25.71(25.25)$ & 24.11 & $0.80(0.80)$ & $0.62(0.61)$ & $12.88(12.37)$ \\
\hline PM6:Y6:PCBSe & $26.99(26.56)$ & 25.72 & $0.81(0.81)$ & $0.63(0.64)$ & $13.97(13.85)$ \\
\hline
\end{tabular}

Average values in parenthesis were calculated from over eight devices. ${ }^{a}$ Calculated $J_{\mathrm{SC}}$ from the integration of EQE spectra.

conditions. $^{38}$ The binary and all ternary devices exhibited a similar $\alpha$ value $(\alpha \sim 0.83)$ indicating that all the devices are affected by a similar degree of bimolecular recombination and that the addition of a third component does not affect this process. Since all devices showed similar alpha values, the differences in the $J_{\mathrm{SC}}$ and $\mathrm{EQE}$ values of binary and ternary OSCs cannot be related to the bimolecular recombination. To gain more insight into the recombination losses, we calculated the ideality factor $\left(n_{\mathrm{id}}\right)$ by fitting the logarithmic $V_{\mathrm{OC}} v$ s. $P_{\text {light }}$ plot (Fig. $\left.3 \mathrm{~b}\right)$ by the equation $V_{\mathrm{OC}} \propto\left(n_{\mathrm{id}} k\right.$ $T / q) \operatorname{Ln}\left(P_{\text {light }}\right)$, where $k$ is the Boltzmann constant, $\mathrm{T}$ is the temperature in kelvin, and $q$ is the elementary charge.

Typically, the $n_{\mathrm{id}}$ value lies in the range $1<n_{\mathrm{id}}<2$; a $n_{\mathrm{id}}$ value equal to unity indicates the presence of bimolecular recombination, while a $n_{\mathrm{id}}$ value higher than 1 indicates that the charge recombination is governed by monomolecular and trap-assisted recombination mechanisms. ${ }^{39,40}$ The $n_{\mathrm{id}}$ values are $1.19,1.09,1.05$ and 1.04 for binary, TOSC-PCBM,-PCBTh and -PCBSe devices, respectively, indicating that the addition of the PCBM derivatives decreases the monomolecular and trapassisted recombination in the devices. These results demonstrated that trap-assisted recombination in PM6:Y6-based OSCs can be minimized by adding a small amount of PCBM as the third component. Importantly, the TOCs with PCBM-derivatives (PCBTh and PCBSe) exhibited the lowest degree of trap-assisted recombination.

\section{Photoluminescence measurements}

Photoluminescence (PL) quenching measurements were performed to analyse the charge transfer behaviour in binary and ternary films. Fig. 4 displays the logarithmic PL spectra of the 
a)

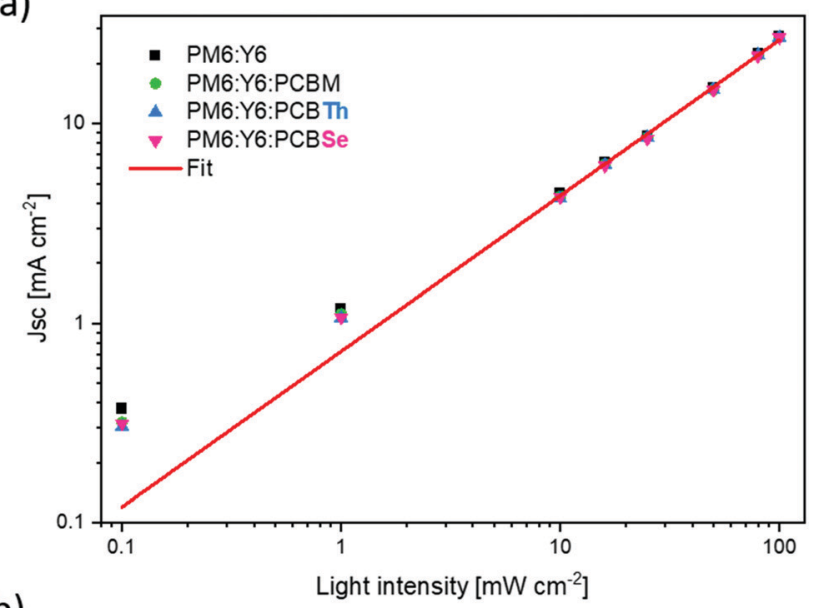

b)

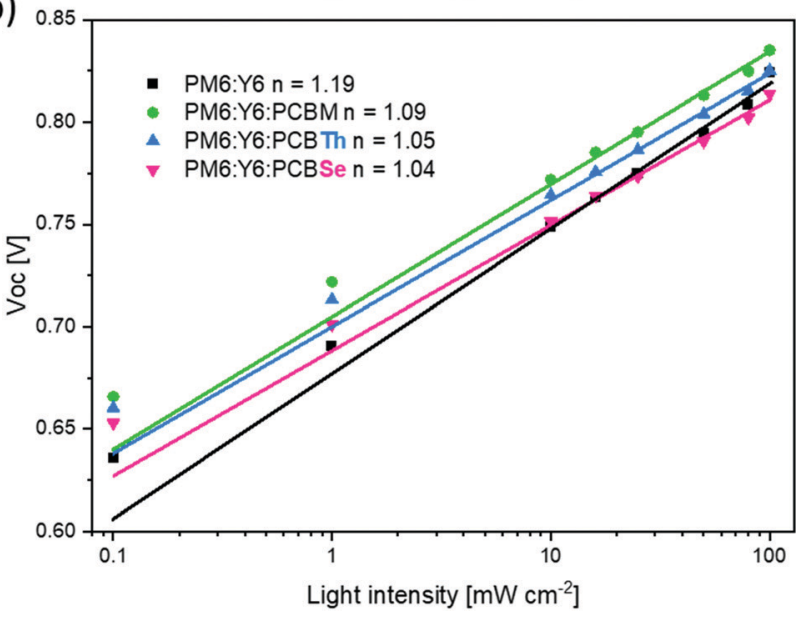

Fig. 3 (a) $J_{S C}$ and (b) $V_{O C}$ light dependence of binary OSCs and ternary OSCs with PCBM, PCBTh, and PCBSe.

neat PM6, binary (PM6:Y6) and ternary (PM6:Y6:PCBMderivatives) films measured with an excitation wavelength of $550 \mathrm{~nm}$, where the neat PM6 film showed the strongest absorption. After adding the different acceptors, the peak at $678 \mathrm{~nm}$, corresponding to the PM6, decreases by one order of magnitude, indicating that there is a charge transfer between the donor PM6 and the different acceptors. The ternary films prepared with PCBM, PCBTh and PCBSe showed a higher quenching degree of the PM6 signal than that of binary PM6:Y6, which proves an enhancement of the charge transfer. Thus, the improvement of the performance parameters of TOSCs with those PCBM-derivatives could be attributed to the better charge transfer. On the other hand, the ternary film with PCBFu showed a lower quenching degree of PM6 than the binary film, indicating that the presence of the furan substituent decreases the charge transfer. This result could explain the lower $J_{\mathrm{SC}}$ and EQE and performance of this TOSC.

\section{Morphological characterization}

To further investigate the effect of the lateral chain substitution on the performance of ternary cells, atomic force microscopy (AFM) characterization has been performed on the samples.

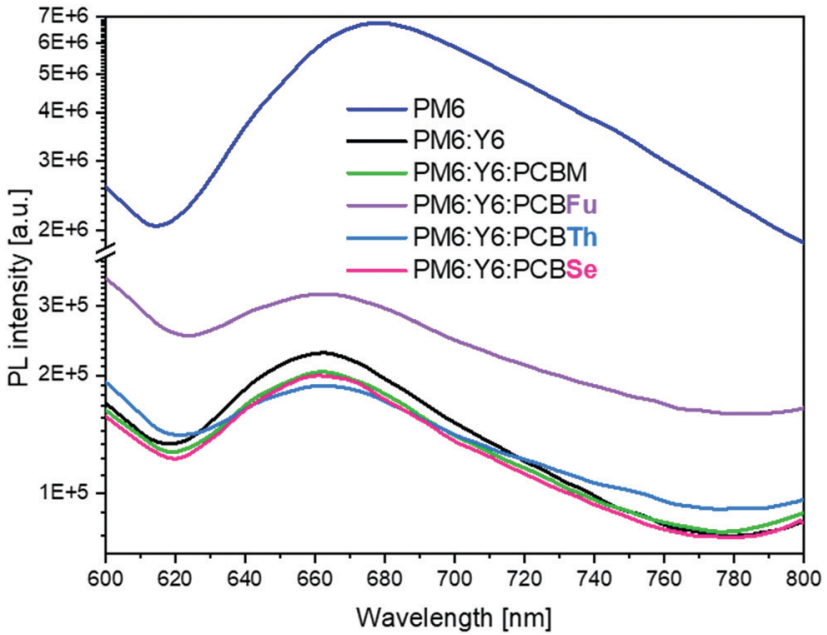

Fig. 4 Photoluminescence spectra of neat PM6, binary PM6:Y6 and ternary blends, PM6:Y6:PCBM-derivatives (PCBM, PCBFu, PCBTh, and PCBSe) after excitation at $550 \mathrm{~nm}$.

Fig. 5 shows the height and phase images of the binary and ternary films deposited on glass. The binary cell PM6:Y6 shows a structure composed of nanofibers, typical of PM6-containing films,${ }^{41}$ with marked phase separation which results in a film roughness of almost $5 \mathrm{~nm}$. The addition of PCBM to the blend reduces the size of the fibrils and the domains thereby decreasing the roughness to $2.03 \mathrm{~nm}$. The addition of the chalcogene containing ligand of PCBM leads to subtle changes in the final morphology of the ternary blends. Furan has little impact in comparison to PCBM. The RMS is similar although the phase distribution is slightly smaller. On the other hand, thiophene gives rise to an increase in the domains and the RMS. The phase distribution allows seeing bigger domains whereas the RMS increases to $2.4 \mathrm{~nm}$. Finally, the selenophene enhances the mixture of the components of the blend, giving rise to the smoothest surface, with an RMS of $1.4 \mathrm{~nm}$, and smaller nanofibrous domains.

To evaluate the vertical phase segregation, water contact angle measurements have been performed on films of neat donor and acceptor materials, and films of the blends (see Table 3). The contact angle values for the neat donor PM6 and acceptor Y6 materials are $99.90 \pm 7.77^{\circ}$ and $96.00 \pm 3.50^{\circ}$, respectively. Regarding the neat PCBM derivatives, the water contact angles are similar pointing to a small influence of the chalcogenide substituent on the variation of the hydrophobicity of the sample. However, there is a small trend following the electronegativity of the atomic substituent, being furan more hydrophilic than selenophene. However, the measurement of the values of the water contact angle on the ternary blends reveals different behaviours depending on the nature of the PCBM derivative. Thus, PCBM shows partial segregation of the three components while in PCBFu there is strong preferential segregation of the PCBFu towards the surface of the photoactive layer. On the other hand, the water contact angle in the PCBTh and PCBSe devices corresponds mostly to the average values of the components suggesting the intermixing of all of them. 
a) PM6:Y6

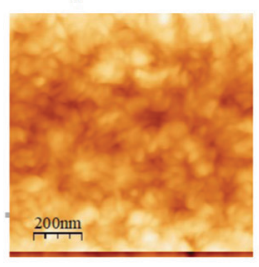

$\mathrm{RMS}=4.80 \mathrm{~nm}$

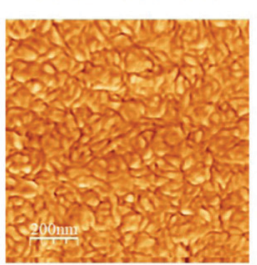

b) PM6:Y6:PCBM

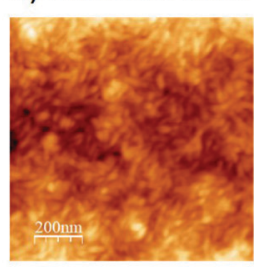

$\mathrm{RMS}=2.03 \mathrm{~nm}$
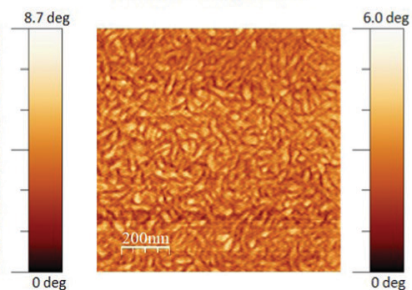

c) PM6:Y6:PCBFu

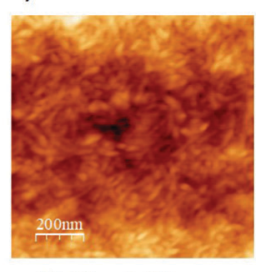

$\mathrm{RMS}=2.02 \mathrm{~nm}$

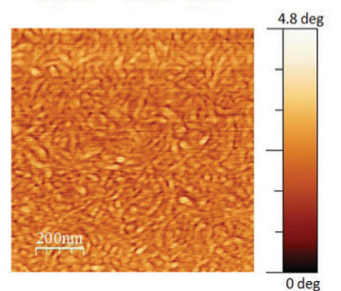

d) PM6:Y6:PCBTh

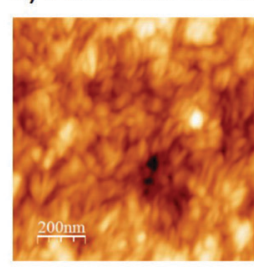

$\mathrm{RMS}=2.38 \mathrm{~nm}$

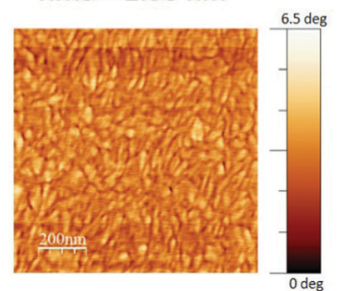

e) PM6:Y6:PCBSe
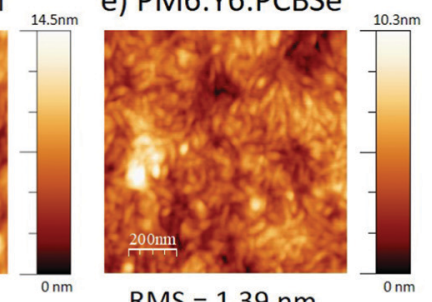

$\mathrm{RMS}=1.39 \mathrm{~nm}$

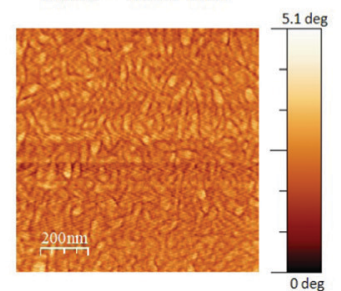

Fig. 5 AFM height (top) and phase (bottom) images of the blend films, (a) PM6:Y6, (b) PM6:Y6:PCBM, (c) PM6:Y6:PCBFu, (d) PM6:Y6:PCBTh and (e) PM6:Y6:PCBSe. The scan size is $1 \times 1 \mu \mathrm{m}$.

Table 3 Contact angle measurements of the water droplet on neat donor and acceptors films, and on ternary blend films

\begin{tabular}{llll}
\hline Neat film & Contact angle $\left[{ }^{\circ}\right]$ & Ternary film & Contact angle $\left[{ }^{\circ}\right]$ \\
\hline PM6 & $99.70 \pm 7.77$ & PM6:Y6:PCBM & $92.33 \pm 5.28$ \\
Y6 & $96.00 \pm 1.45$ & PM6:Y6:PCBFu & $89.54 \pm 0.94$ \\
PCBM & $89.98 \pm 1.77$ & PM6:Y6:PCBTh & $98.60 \pm 0.94$ \\
PCBFu & $90.05 \pm 1.45$ & PM6:Y6:PCBSe & $94.84 \pm 4.70$ \\
PCBTh & $90.39 \pm 1.84$ & & \\
PCBSe & $92.06 \pm 2.37$ & &
\end{tabular}

Contact angle values were averaged over 4 measurements at 4 different positions for each sample.

We have also performed DSC measurements of the PCBX derivatives (Fig. S28, ESI $\dagger$ ). The thermogram of PCBM reveals three endothermic peaks at $250^{\circ}$, corresponding to the crystallization temperature and at 276 and $284{ }^{\circ} \mathrm{C}$ for the melting point. PCBFu shows undefined peaks between 180 and $250{ }^{\circ} \mathrm{C}$ suggesting the amorphous nature of the molecule, whereas PCBTh posseses a single endothermic peak at $269{ }^{\circ} \mathrm{C}$. Finally, PCBSe shows a melting temperature of $299{ }^{\circ} \mathrm{C}$. The thermograms of the binary blends (Fig. S29, ESI $\dagger$ ) show two endothermic peaks at 250.7 and $296.3{ }^{\circ} \mathrm{C}$, assigned to the melting points of Y6. Upon addition of the ternary component, only the peak associated with the Y6 is clearly visible, due to the low concentration of the ternary component in the blend. Moreover, the melting temperatures of the blends decrease as compared to those of the binary blend (Table S1, ESI $\dagger$ ). This is assigned to the modification of the crystallinity and the molecular arrangement in the active layer. The shift in the melting temperature is proportional to the performance of the devices. On the other hand, the decrease in the enthalpy of the peak associated with the melting temperature of Y6 suggests that the aggregation of the acceptor molecule has been restrained. Again, a higher decrease is associated with higher performance. This is in agreement with the AFM measurements, where the RMS is lowered upon the addition of the ternary component demonstrating that an optimized mixture of the components is key to achieve higher efficiencies.

\section{Conclusions}

Ternary cells have been prepared combining PCBM chalcogen derivatives with the PM6:Y6 host. The nature of the chalcogen has a strong effect on the performance of the devices. Thus, the furan substituent, although enhances the FF, allows for lower $J_{\text {SC }}$ and $V_{\text {OC }}$ arising from a higher leakage current probably due to the low affinity with the other components of the blend, resulting in strong phase segregation. The rest of the PCBM derivatives do not affect the rate of bimolecular recombination but decrease the trap assisted recombination. This is translated to a higher $\mathrm{FF}$ and $V_{\mathrm{OC}}$ and a lower leakage current in comparison with those of the binary blend and is assigned to the partial mixing of the three components that also favours charge transfer. Finally, the PCBTh derivative offers the best results, especially because of higher $J_{\mathrm{SC}}$ when compared to the other derivatives. The optimized phase segregation and mixing of the three components facilitate the charge separation and reduce the trap assisted recombination which results in an increase of the FF giving the highest PCE of $14.6 \%$.

This study highlights the effect of fullerene acceptors containing thiophene and selenophene rings as a promising approach to improve the efficiency of ternary OSCs. Moreover, it opens the way to further enhance the efficiency of the devices if more efficient fullerenes such as PC71BM are applied.

\section{Experimental}

\section{Synthesis of PCBM-derivatives}

The details of the entire synthetic procedure and characterization data are provided in the ESI. $\dagger$ 


\section{Device fabrication}

Patterned indium tin oxide (ITO) glass substrates (15 $\Omega / \mathrm{sq}$ ) were cleaned with an alkaline cleaning concentrate (Hellmanex) diluted in deionized water, acetone, methanol and isopropanol in an ultrasonic bath for 15 min consecutively. The ITO-substrates were dried under dry nitrogen and then treated in a UV-Ozone cleaner for $20 \mathrm{~min}$. The poly(3,4-ethylenedioxythiophene) polystyrene sulfonate (PEDOT:PSS) as a hole transporting material was spin-coated on the patterned ITO glass substrates at $4000 \mathrm{rpm}$ for $40 \mathrm{~s}$ and then annealed at $150{ }^{\circ} \mathrm{C}$ for $10 \mathrm{~min}$ under an air atmosphere. After annealing, the substrates were transferred to a glovebox filled with nitrogen. The binary PM6:Y6 $(1: 1.2 \mathrm{w} / \mathrm{w})$ and ternary PM6:Y6:PCBM-derivative (PCBM, PCBFu, PCBTh, PCBSe, 1:1: $0.2 \mathrm{w} / \mathrm{w}$ ) solutions were prepared by dissolving the donor and acceptors materials in chloroform and 1-chloronaphthalene dry solvents (99.5: $0.5 \mathrm{v} / \mathrm{v})$. The concentration of PM6 was $7 \mathrm{mg} \mathrm{mL}$ for all the solutions. The binary and all the ternary solutions were spin-coated on the PEDOT:PSS layer at $1800 \mathrm{rpm}$ for $40 \mathrm{~s}$. Then, the samples were annealed at $100{ }^{\circ} \mathrm{C}$ for $10 \mathrm{~min}$. The PDINO as an electron transporting material (ETM) was dissolved in anhydrous methanol $\left(1 \mathrm{mg} \mathrm{mL}^{-1}\right)$. PDINO solution was spin-coated on active layers at $3000 \mathrm{rpm}$ for $40 \mathrm{~s}$. Finally, $100 \mathrm{~nm}$ of aluminum (Al) as the metal cathode were thermally evaporated on top of the active layer through a shadow mask with an effective area of $9 \mathrm{~mm}^{2}$.

\section{Characterization}

Ultraviolet and visible (UV-Vis). The UV-Vis measurements were carried out using an Agilent Cary 60 UV-Vis spectrophotometer with two silicon detectors, double beam optics, a Czerny-Turner monochromator, 190-1100 nm range and the light source is a xenon flash lamp.

Photovoltaics measurements. All $J-V$ characteristics were determined in the forward voltage sweep direction from -1 to $1 \mathrm{~V}$ at room temperature under dark and illumination conditions. The illumination measurements were carried out using a solar simulator (Abet Technologies model 11000 class type A, Xenon arc) and a Keithley 2400 source-measurement unit. The light intensity was calibrated using a NREL certified monocrystalline silicon photodiode. The $J-V$ measurements with different light intensities were obtained using different optical filters.

External quantum efficiency measurements. EQE data were recorded using quantum efficiency measurement systems from Lasing, S.A. (IPCE-DC, LS1109-232) and a Newport 2936-R power-meter unit. The EQE measurements were taken under a wavelength sweep from $300 \mathrm{~nm}$ to $1000 \mathrm{~nm}$.

Photoluminescence measurements (PL). The fluorescence spectra were recorded in the range of 600-800 $\mathrm{nm}$ with an excitation wavelength of $550 \mathrm{~nm}$ using a spectrofluorimeter Fluorolog from Horiba Jobin Yvon Lrd with PMT and InGaAs detectors.

Atomic force microscopy measurements. AFM was performed in tapping mode on a molecular imaging model Pico SPM II (pico+). Images were recorded in air using silicon probes with a radius of 2-3 $\mathrm{nm}$ and at a resonant frequency of $150 \mathrm{kHz}$.
Contact angle measurements. The contact angles of PM6, Y6, and PCBM derivative films, as well as binary PM6:Y6 and ternary PM6:Y6:PCBM-derivative films were measured by using an optical tensiometer (Attension ${ }^{\circledR}$ Theta Lite), using the sensile drop technique. The measurements were carried out under ambient conditions by applying water droplets of $10 \mu \mathrm{L}$. The contact angle values were averaged over 4 measurements at 4 different positions for each film.

\section{Author contributions}

A. C. E synthesized and characterized the PCBFu, PCBTh and PCBSe acceptors under the supervision of J. L. D. J. G. S. fabricated and characterized the binary and ternary devices under the supervision of E. M. F and E. P. All authors have discussed the results and participated in the writing of this manuscript.

\section{Conflicts of interest}

There are no conflicts to declare.

\section{Acknowledgements}

J. G. S., E. M. F. and E. P. thank MINECO (project PID2019109389RB-I00) and SGR-AGAUR 2017SGR00978. E. P. is also thankful to ICIQ, CERCA, and ICREA for financial support. A. C. E. and J. L. D. acknowledges Ikerbasque, the Basque Foundation for Science, for an "Ikerbasque Research Associate" contract, the Polymat Foundation and the MICINN of Spain for RED2018-102815-T and RTI2018-101782-B-I00 grants.

\section{References}

1 G. Yu, J. Gao, J. C. Hummelen, F. Wudl and A. J. Heeger, Science, 1995, 270, 1789-1791.

2 G. Dennler, M. C. Scharber and C. J. Brabec, Adv. Mater., 2009, 21, 1323-1338.

3 Y. Liu, N. Qi, T. Song, M. Jia, Z. Xia, Z. Yuan, W. Yuan, K. Q. Zhang and B. Sun, ACS Appl. Mater. Interfaces, 2014, 6, 20670-20675.

4 Q. Liu, Y. Jiang, K. Jin, J. Qin, J. Xu, W. Li, J. Xiong, J. Liu, Z. Xiao, K. Sun, S. Yang, X. Zhang and L. Ding, Sci. Bull., 2020, 65, 272-275.

5 Y. Lin, Y. Firdaus, F. H. Isikgor, M. I. Nugraha, E. Yengel, G. T. Harrison, R. Hallani, A. El-Labban, H. Faber, C. Ma, X. Zheng, A. Subbiah, C. T. Howells, O. M. Bakr, I. McCulloch, S. De Wolf, L. Tsetseris and T. D. Anthopoulos, ACS Energy Lett., 2020, 5, 2935-2944.

6 J. Hou, O. Inganas, R. H. Friend and F. Gao, Nat. Mater., 2018, 17, 119-128.

7 Z. Zheng, H. Yao, L. Ye, Y. Xu, S. Zhang and J. Hou, Mater. Today, 2020, 35, 115-130. 
8 X. Zhan, Z. Tan, B. Domercq, Z. An, X. Zhang, S. Barlow, Y. Li, D. Zhu, B. Kippelen and S. R. Marder, J. Am. Chem. Soc., 2007, 129, 7246-7247.

9 M. Z. Abdul Aziz, K. Higashimine, N. Shioya, T. Shimoaka, T. Hasegawa, H. Sakai, V. Vohra and H. Murata, RSC Adv., 2020, 10, 37529-37537.

10 Y. He and Y. Li, Phys. Chem. Chem. Phys., 2011, 13, 1970-1983.

11 Y. Lin, J. Wang, Z. G. Zhang, H. Bai, Y. Li, D. Zhu and X. Zhan, Adv. Mater., 2015, 27, 1170-1174.

12 B. Guo, W. Li, X. Guo, X. Meng, W. Ma, M. Zhang and Y. Li, Adv. Mater., 2017, 29, 1-7.

13 G. Forti, A. Nitti, P. Osw, G. Bianchi, R. Po and D. Pasini, Int. J. Mol. Sci., 2020, 21, 1-16.

14 H. Sun, F. Chen and Z. K. Chen, Mater. Today, 2019, 24, 94-118.

15 S. Li, C.-Z. Li, M. Shi and H. Chen, ACS Energy Lett., 2020, 5, 1554-1567.

16 J. Yuan, Y. Zhang, L. Zhou, G. Zhang, H. L. Yip, T. K. Lau, X. Lu, C. Zhu, H. Peng, P. A. Johnson, M. Leclerc, Y. Cao, J. Ulanski, Y. Li and Y. Zou, Joule, 2019, 3, 1140-1151.

17 Q. Guo, Q. Guo, Y. Geng, A. Tang, M. Zhang, M. Du, X. Sun and E. Zhou, Mater. Chem. Front., 2021, 5, 3257-3280.

18 Z. C. Wen, H. Yin and X. T. Hao, Surf. Interfaces, 2021, 23, 100921.

19 Q. Ma, Z. Jia, L. Meng, J. Zhang, H. Zhang, W. Huang, J. Yuan, F. Gao, Y. Wan, Z. Zhang and Y. Li, Nano Energy, 2020, 78, 105272.

20 N. Gasparini, S. H. K. Paleti, J. Bertrandie, G. Cai, G. Zhang, A. Wadsworth, X. Lu, H.-L. Yip, I. McCulloch and D. Baran, ACS Energy Lett., 2020, 5, 1371-1379.

21 W. Tang, W. Peng, M. Zhu, H. Jiang, W. Wang, H. Xia, R. Yang, O. Inganäs, H. Tan, Q. Bian, E. Wang and W. Zhu, J. Mater. Chem. A, 2021, 9, 20493-20501.

22 H. Dahiya, V. Cuesta, P. De La Cruz, F. Langa and G. D. Sharma, ACS Appl. Energy Mater., 2021, 4, 4498-4506.

23 M. Privado, H. Dahiya, P. de la Cruz, M. L. Keshtov, F. Langa and G. D. Sharma, J. Mater. Chem. C, 2021, 9, 16272-16281.

24 T. Yan, W. Song, J. Huang, R. Peng, L. Huang and Z. Ge, Adv. Mater., 2019, 31, 1-8.
25 M. A. Pan, T. K. Lau, Y. Tang, Y. C. Wu, T. Liu, K. Li, M. C. Chen, X. Lu, W. Ma and C. Zhan, J. Mater. Chem. A, 2019, 7, 20713-20722.

26 G. Y. Ge, W. Xiong, K. K. Liu, H. S. Ryu, S. S. Wan, B. Liu, A. Mahmood, H. R. Bai, J. F. Wang, Z. Wang, H. Y. Woo, Y. Sun and J. L. Wang, J. Mater. Chem. C, 2021, 9, 1923-1935.

27 Y. Chen, J. Liang, Y. Yu, L. Wang, C. Weng and P. Shen, Org. Electron., 2021, 89, 106015.

28 Suman, A. Bagui, R. Datt, V. Gupta and S. P. Singh, Chem. Commun., 2017, 53, 12790-12793.

29 L. M. Popescu, P. Van'T Hof, A. B. Sieval, H. T. Jonkman and J. C. Hummelen, Appl. Phys. Lett., 2006, 89, 3-6.

30 Y. C. Lai, T. Higashihara, J. C. Hsu, M. Ueda and W. C. Chen, Sol. Energy Mater. Sol. Cells, 2012, 97, 164-170.

31 O. Fernandez-Delgado, E. Castro, C. R. Ganivet, K. Fosnacht, F. Liu, T. Mates, Y. Liu, X. Wu and L. Echegoyen, ACS Appl. Mater. Interfaces, 2019, 11, 34408-34415.

32 Y. He, G. Zhao, B. Peng and Y. Li, Adv. Funct. Mater., 2010, 20, 3383-3389.

33 J. Yao, B. Qiu, Z. G. Zhang, L. Xue, R. Wang, C. Zhang, S. Chen, Q. Zhou, C. Sun, C. Yang, M. Xiao, L. Meng and Y. Li, Nat. Commun., 2020, 11, 1-10.

34 C. Yang, J. Zhang, N. Liang, H. Yao, Z. Wei, C. He, X. Yuan and J. Hou, J. Mater. Chem. A, 2019, 7, 18889-18897.

35 X. Wang, Q. Sun, J. Gao, X. Ma, J. H. Son, S. Y. Jeong, Z. Hu, L. Niu, H. Y. Woo, J. Zhang and F. Zhang, Sol. RRL, 2021, 5, 1-9.

36 D. Huang, Y. Li, Z. Xu, S. Zhao, L. Zhao and J. Zhao, Phys. Chem. Chem. Phys., 2015, 17, 8053-8060.

37 J. D. Servaites, M. A. Ratner and T. J. Marks, Energy Environ. Sci., 2011, 4, 4410-4422.

38 D. Credgington, Y. Kim, J. Labram, T. D. Anthopoulos and J. R. Durrant, J. Phys. Chem. Lett., 2011, 2, 2759-2763.

39 S. R. Cowan, A. Roy and A. J. Heeger, Phys. Rev. B: Condens. Matter Mater. Phys., 2010, 82, 1-10.

40 T. Kirchartz and J. Nelson, Phys. Rev. B: Condens. Matter Mater. Phys., 2012, 86, 1-12.

41 F. Du, H. Wang, Z. Zhang, L. Yang, J. Cao, J. Yu and W. Tang, Mater. Horiz., 2021, 8, 1008-1016. 